\title{
Efficacy of trifluridine and tipiracil (TAS-102) versus placebo, with supportive care, in a randomized, controlled trial of patients with metastatic colorectal cancer from Spain: results of a subgroup analysis of the phase 3 RECOURSE trial
}

\author{
F. Longo-Muñoz ${ }^{1}$ G. Argiles ${ }^{2} \cdot$ J. Tabernero ${ }^{2} \cdot$ A. Cervantes $^{3}$ - C. Gravalos ${ }^{4}$ \\ C. Pericay ${ }^{5} \cdot$ S. Gil-Calle ${ }^{6} \cdot$ H. Mizuguchi ${ }^{7} \cdot$ A. Carrato-Mena ${ }^{1} \cdot$ M. L. Limón ${ }^{8}$. \\ R. Garcia-Carbonero ${ }^{4}$
}

Received: 2 March 2016/Accepted: 20 June 2016/Published online: 21 July 2016

(c) Federación de Sociedades Españolas de Oncología (FESEO) 2016

\begin{abstract}
Purpose TAS-102 is a combination of the thymidine-based nucleoside analog trifluridine and the thymidine phosphorylase inhibitor tipiracil. Efficacy and safety of TAS-102 in patients with metastatic colorectal cancer (mCRC) refractory or intolerant to standard therapies were evaluated in the phase 3 RECOURSE trial. Results of RECOURSE demonstrated significant improvement in overall survival (OS) and progression-free survival (PFS) with TAS-102 versus placebo [hazard ratio $(\mathrm{HR})=0.68$ and 0.48 for $\mathrm{OS}$ and PFS, respectively; both $P<0.001]$. The current analysis evaluates efficacy and safety of TAS-102 in the RECOURSE Spanish subgroup.

Methods Primary and key secondary endpoints were evaluated in a post hoc analysis of the RECOURSE Spanish subgroup, using univariate and multivariate analyses. Safety and tolerability were reported with descriptive statistics.
\end{abstract}

On behalf of the RECOURSE Study Group.

R. Garcia-Carbonero

rgcarbonero@gmail.com

1 Hospital Universitario Ramón y Cajal, Servicio Oncología Médica, (Center Affiliated with the Red Tematica de Investigacion Cooperativa en Cancer, Instituto Carlos III, Spanish Ministry of Science and Innovation), Carretera de Colmenar Viejo Km 9.100, Madrid, Spain 28034

2 Hospital Universitario Vall d'Hebron, Universitat Autònoma de Barcelona, (Center Affiliated with the Red Tematica de Investigacion Cooperativa en Cancer, Instituto Carlos III, Spanish Ministry of Science and Innovation), P. Vall d'Hebron 119-129, 08035 Barcelona, Spain

3 Biomedical Research Institute INCLIVA, University of Valencia, Av. Menéndez Pelayo 4 accesorio, 46010 Valencia, Spain
Results The RECOURSE Spanish subgroup included 112 patients (mean age 61 years, $62 \%$ male). Median OS was 6.8 months in the TAS-102 group $(n=80)$ versus 4.6 months in the placebo group $(n=32)[\mathrm{HR}=0.47$; $95 \%$ confidence interval (CI): $0.28-0.78 ; P=0.0032$ ). Median PFS was 2.0 months in the TAS-102 group and 1.7 months in the placebo group (HR $=0.47 ; 95 \% \mathrm{CI}$ : $0.30-0.74 ; P=0.001)$. Eighty $(100 \%)$ TAS- 102 versus 31 $(96.9 \%)$ placebo patients had adverse events (AEs). The most common drug-related $\geq$ Grade $3 \mathrm{AE}$ was neutropenia (40\% TAS-102 versus $0 \%$ placebo). There was $1(1.3 \%)$ case of febrile neutropenia in the TAS-102 group versus none in the placebo group.

Conclusions In the RECOURSE Spanish subgroup, TAS102 was associated with significantly improved OS and PFS versus placebo, consistent with the overall RECOURSE population. No new safety signals were identified.

ClinicalTrials.gov study number NCT01607957

4 Hospital Universitario Doce de Octubre, Servicio Oncología Médica, (Center Affiliated with the Red Tematica de Investigacion Cooperativa en Cancer, Instituto Carlos III, Spanish Ministry of Science and Innovation), Avenida Cordoba km 5.4, Madrid, Spain 28041

5 Hospital Universitario de Sabadell, Corporació Sanitària Parc Tauli, Parc Taulí, 1, 08208 Sabadell, Spain

6 Hospital Carlos Haya, Av. de Carlos Haya, s/n, 29010 Málaga, Spain

7 Taiho Oncology Inc, 202 Carnegie Center, Suite 100, Princeton, NJ 08540, USA

8 Hospital Universitario Virgen del Rocío, $S^{\circ}$ Oncologia Medica, Manuel Siurot, 41013 Seville, Spain 
Keywords TAS-102 - Metastatic colorectal cancer . Trifluridine - Tipiracil hydrochloride · Fluoropyrimidine . Spain

\section{Introduction}

TAS-102 is an oral combination treatment consisting of trifluridine (FTD), a thymidine-based nucleoside analog, and tipiracil hydrochloride (TPI), at a molar ratio of 1:0.5 (weight ratio: 1:0.471). It is approved for use in Japan and the United States, and also has been recently approved in Europe. The primary cytotoxic mechanism of oral FTD is through incorporation into DNA following phosphorylation by thymidine kinase, leading to DNA dysfunction (Fig. 1) $[1,2]$. This mechanism is distinct from that of 5-fluorouracil (5-FU) and other fluoropyrimidines that produce their cytotoxic effects through inhibition of thymidylate synthase. Although phosphorylated FTD does inhibit this enzyme, this activity is secondary to its direct effects on DNA when administered orally. This may explain the activity of TAS-102 in human cancer xenografts resistant to 5-FU $[1,3]$. TPI improves the bioavailability of FTD by inhibiting its catabolism by thymidine phosphorylase [4]. The combination of these two agents makes TAS-102 an attractive candidate for treatment of patients with metastatic colorectal cancer (mCRC) who are refractory to fluoropyrimidines [5].

TAS-102 has shown promise in a number of phase 1 and 2 trials [6-10]. A phase 2, double-blind, randomized, controlled trial conducted in Japanese patients with mCRC refractory to 5-FU, irinotecan, and oxaliplatin demonstrated a median overall survival (OS) of 9.0 months with TAS-102 compared with 6.6 months in the placebo group [hazard ratio $(\mathrm{HR})=0.56 ; 95 \%$ confidence interval $(\mathrm{CI})$, $0.39-0.81 ; P=0.001]$. More recently, in the phase 3 RECOURSE trial in patients with mCRC refractory to standard therapies, TAS-102 demonstrated a significant improvement compared with placebo in median OS (7.1 vs 5.3 months; HR $=0.68 ; 95 \%$ CI, 0.58-0.81; $P<0.001)$ and progression-free survival (PFS) (2.0 vs 1.7 months; $\mathrm{HR}=0.48 ; 95 \% \mathrm{CI}, 0.41-0.57 ; P<0.001)$ [11].

The initial phase 2 trial of TAS-102 included only patients from Japan, and a subset of patients enrolled in RECOURSE were from Europe [9, 11]. It is of interest, therefore, to assess the efficacy of TAS-102 in patients enrolled in Spain, which accounted for a sizeable portion $(14 \%)$ of patients enrolled in the international RECOURSE trial. The objective of the current analysis was to evaluate the efficacy and safety of TAS-102 in the Spanish subgroup of patients enrolled in the RECOURSE trial.

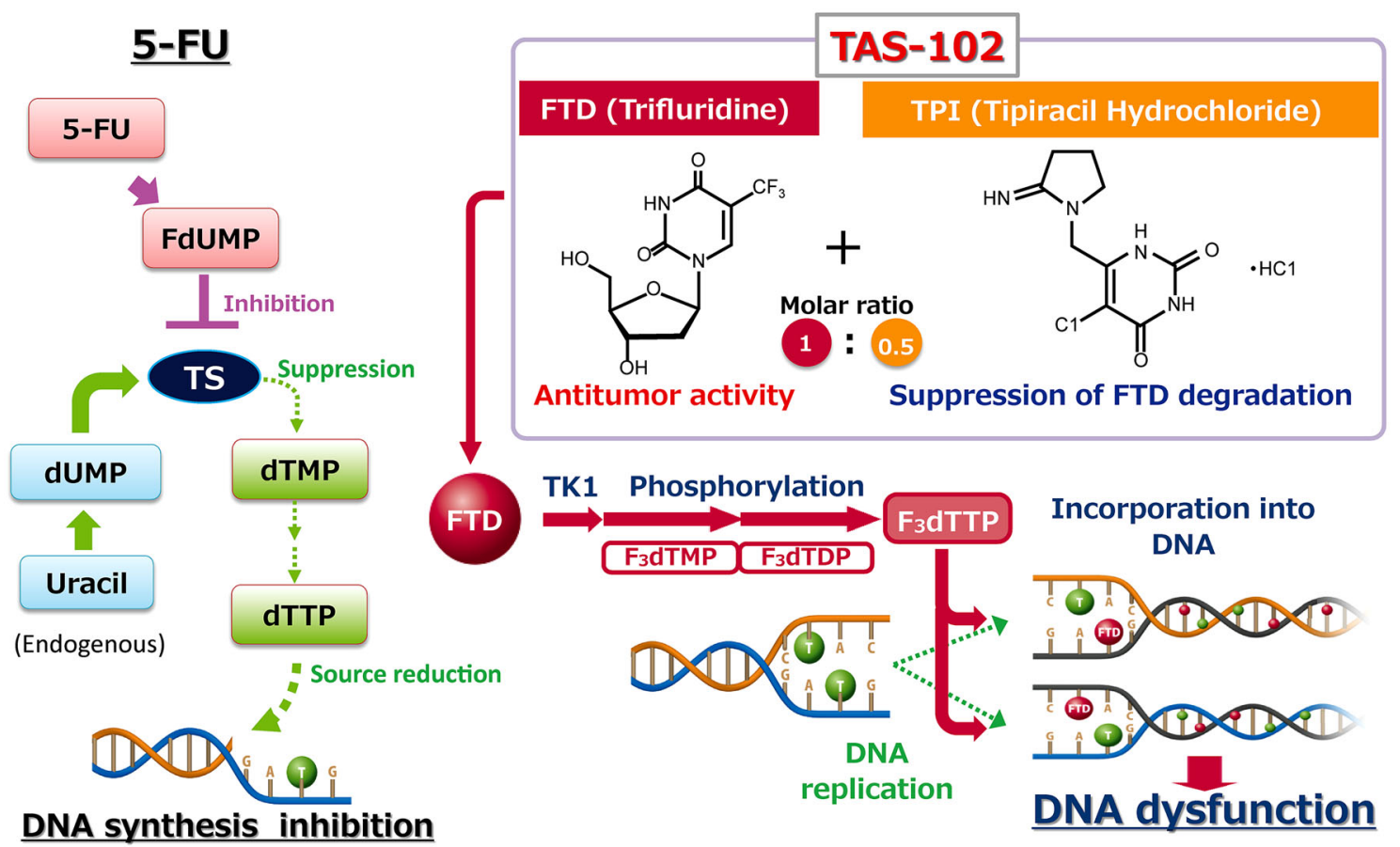

Fig. 1 Comparison of the mechanisms of action of TAS-102 and 5-FU. 5-FU fluorouracil; $d T M P$ and $d T T P$ deoxythymidine mono- and triphosphate; $d U M P$ deoxyuridine monophosphate; $F_{3} d T M P, F_{3-}$ $d T D P$, and $F_{3} d T T P$ trifluorodeoxythymidine mono-, di-, and triphosphate; FdUMP fluorodeoxyuridine monophosphate; TK1 thymidine kinase 1; TPase thymidine phosphorylase; TPI tipiracil hydrochloride; $T S$ thymidylate synthase 


\section{Methods and patients}

\section{Study design}

The protocol for the RECOURSE study has been described in detail previously [11]. Briefly, the RECOURSE trial was a global, multicenter, randomized, double-blind, placebocontrolled phase 3 trial comparing TAS-102 plus best supportive care with placebo plus best supportive care. Patients were randomly assigned in a 2:1 ratio to receive TAS-102 or placebo and were stratified according to KRAS status (wild type, mutant status), time since diagnosis of first metastasis ( $<18$ months, $\geq 18$ months), and geographic region [Japan or Western (United States, Europe, and Australia combined)]. This analysis focuses on those patients in the European stratum who were randomized in Spain. Subjects were randomized at 11 different centers in Spain. This trial is registered with ClinicalTrials.gov number, NCT01607957.

The study was conducted according to the principles of the Declaration of Helsinki and the International Council for Harmonisation Good Clinical Practice Guidelines, and the study was approved by institutional review boards at each participating center. All patients provided written informed consent.

\section{Patients}

Eligible patients with metastatic, biopsy-documented adenocarcinoma of the colon or rectum who had received $\geq 2$ prior lines of therapy with standard chemotherapies and had been refractory to antitumor therapy were eligible for randomization. Prior therapy could have included adjuvant treatment if tumor recurrence had occurred within 6 months, tumor progression within 3 months after the last administration of therapy, or if clinical adverse events precluded rechallenge with standard chemotherapy. Knowledge of KRAS status was required, and patients must have received prior chemotherapy with a fluoropyrimidine, oxaliplatin, irinotecan, and bevacizumab, and cetuximab or panitumumab if they had KRAS wild-type tumors. In addition, patients had to be $\geq 18$ years of age, have adequate bone-marrow, liver, and renal function, and have an Eastern Cooperative Oncology Group (ECOG) performance status of 0 or 1 . Patients were centrally randomized via an Interactive Voice/Web Response System based on a dynamic allocation method.

\section{Endpoints}

The primary endpoint was OS, which was defined as the time from randomization to death from any cause.
Secondary endpoints included PFS, which was defined as the time from randomization to the first radiographic confirmation of disease progression or death from any cause, overall response rate (the proportion of patients with a complete or partial response), disease control rate (the proportion of patients with a complete or partial response, or stable disease, with stable disease assessed at least 6 weeks after starting treatment), time to deterioration of ECOG performance status to 2 or greater, and safety. Radiographic assessments were conducted every 8 weeks, and treatment was continued until disease progression as defined by RECIST (version 1.1) [12], clinical progression, development of serious adverse events, study withdrawal, death, or a decision by the physician that discontinuation was in the patient's best interest. Adverse events were classified and graded according to the National Cancer Institute Common Terminology Criteria for Adverse Events, version 4.03 .

\section{Statistical analysis}

The study protocol included a prespecified analysis of outcomes and safety according to geographic subregion. The same methodology was used for this post hoc analysis of the Spanish subgroup. OS and PFS were analyzed in the intent-to-treat population with the use of a two-sided, stratified log-rank test, with the HR and two-sided $95 \%$ confidence intervals based on a stratified Cox proportional hazards model and the associated Kaplan-Meier survival estimates. The median follow-up time for survival was calculated by means of the reverse Kaplan-Meier method. Objective response and disease control rates were compared using the Fisher's exact test in the subgroup of patients in the intent-to-treat population who had measurable disease at baseline. The time to deterioration of ECOG performance status to 2 (defined as: ambulatory and capable of all self-care but unable to carry out any work activities; up and about more than $50 \%$ of waking hours) was analyzed with a similar approach to that employed for analysis of OS. Adverse events and laboratory abnormalities were summarized for all patients who received at least one dose of study drug. The number of hospitalizations, number and percentage of patients hospitalized, reason for hospitalization, and the total duration of hospitalization were summarized descriptively by treatment group.

\section{Results}

\section{Patients}

Of the 112 patients randomized to the RECOURSE study in Spain, 80 were in the TAS-102 arm and 32 in the 
placebo arm. The Spanish subgroup, therefore, represents $14 \%$ of the overall RECOURSE population and $28 \%$ of the prespecified European stratum. Patient and tumor characteristics for subjects enrolled and randomized in Spain are shown in Table 1, along with characteristics for the overall population. Because patients enrolled in Spain represented a significant portion of the overall and prespecified European stratum, baseline characteristics were well balanced between the two arms. However, there were some differences between the Spanish and overall populations: the Spanish subgroup was entirely Caucasian vs $57.6 \%$ of the overall population; Spanish patients had worse ECOG performance status with $68.8 \%$ having a performance status of $1 \mathrm{vs} 44.0 \%$ in the overall population (31.3 and $56.0 \%$ had performance status of 0 in the two populations, respectively); and more patients in the Spanish subgroup had had prior regorafenib treatment (25.9 vs $18.0 \%)$.

\section{Efficacy}

The median OS in the Spanish subgroup was 6.8 months in the TAS-102 group, significantly greater than the 4.6 months observed in the placebo group $(\mathrm{HR}=0.47$; $95 \%$ CI, 0.28-0.78; $P=0.0032$ ) (Fig. 2a; Table 2). The 1-year OS rate was $27.5 \%$ (95\% CI, 14.0-42.9) in the TAS-102 arm and $20.4 \%$ (95\% CI, 7.9-36.9) in the placebo arm (Fig. 2b). Similarly, median PFS in the TAS-102 group from in Spanish patients was 2.0 months, significantly longer than 1.7 months observed in the placebo group $(\mathrm{HR}=0.47 ; \quad 95 \% \mathrm{CI}, \quad 0.30-0.74 ; \quad P<0.001)$ (Fig. 3; Table 2). As shown in Figs. 2, 3, and Table 2, these results were consistent with the OS and PFS reported for the overall population.

There was no significant difference between arms in terms of the best overall response rate and the disease control rate in the Spanish subgroup. No patients in either group had a complete response. The TAS-102 arm had a partial response rate of $3.9 \%$ (3 patients) vs $0 \%$ in the placebo arm. The disease control rate was 39.5 and $19.4 \%$ in the TAS-102 and placebo arms, respectively. The median time to deterioration of ECOG performance status to 2 was 5.4 months for the TAS-102 group versus 3.3 months for the placebo group (HR $=0.31 ; 95 \% \mathrm{CI}$, $0.18-0.54 ; P<0.0001)$. These results were consistent with response and disease control rates in the overall population.

\section{Safety and tolerability}

Overall Grade $\geq 3$ adverse events in the Spanish subgroup, regardless of causation, occurred in $72.5 \%$ of patients in the TAS- 102 arm and $56.3 \%$ in the placebo arm. The most common nonhematologic adverse events of any grade seen with TAS-102 in $\geq 30 \%$ of the Spanish subgroup were asthenia, nausea, decreased appetite, and diarrhea (Table 3). Asthenia and back pain were the most common $(\geq 5 \%)$ nonhematologic Grade $\geq 3$ adverse events seen with TAS-102. The incidence of asthenia and mucosal inflammation was somewhat higher among Spanish patients than in the overall population and the incidence of fatigue was somewhat lower, but there were no other notable differences in the incidence of individual adverse events.

There were similar incidences of laboratory abnormalities in the Spanish population compared with the overall population. For Grade $\geq 3$ laboratory abnormalities, myelosuppression was common with TAS-102, with 40 and $16.3 \%$ experiencing neutropenia or leukopenia, compared with no patients in the placebo arm; in addition, $13.8 \%$ experienced anemia, compared with $6.3 \%$ in the placebo arm. Lymphocytopenia and thrombocytopenia were also higher in the TAS-102 arm compared with the placebo arm. Total bilirubin and alkaline phosphatase were elevated in a substantial portion of the Spanish population in both treatment groups (Table 3).

Importantly, the rate of serious adverse events and hospitalizations was lower in the TAS-102 arm than in the placebo arm: 20 and $37.5 \%$ of patients experienced serious adverse events in the TAS-102 and placebo arms, respectively. A similar proportion of patients were hospitalized during the study, with the vast majority being due to serious adverse events; one patient in the TAS-102 arm was hospitalized due to febrile neutropenia and one patient in the placebo arm was hospitalized due to health deterioration (Table 3). Overall, TAS-102 was considered to be well tolerated in the Spanish population.

\section{Discussion}

The results observed in the Spanish subgroup (14\% of the total study population) were consistent with the results of the overall RECOURSE study. Indeed, TAS-102 was associated with significant improvements in OS and PFS in the Spanish population. In Spanish patients, the risk of death and risk of disease progression or death were both reduced by $53 \%$ with TAS-102 compared with placebo ( $P=0.0032$ and $P=0.001$, respectively). The reduction in the risk of death with TAS-102 in this population was greater than that observed in the overall population, with an HR of 0.47 (95 \% CI: 0.28-0.78) in the Spanish group and $0.68(0.58-0.81)$ in the overall population [11]. The HR of OS in the Spanish subgroup was lower than that of the overall RECOURSE population. This may be explained by the fact that the Spanish population had a substantially higher percentage of patients with an ECOG status of 1 
Table 1 Patient demographics and baseline characteristics [11]

\begin{tabular}{|c|c|c|c|c|}
\hline & \multicolumn{2}{|l|}{ Spanish subgroup } & \multicolumn{2}{|l|}{ Overall population } \\
\hline & TAS-102 $(n=80)$ & Placebo $(n=32)$ & TAS-102 $(n=534)$ & Placebo $(n=266)$ \\
\hline Gender, male, $n(\%)$ & $48(60.0)$ & $21(65.6)$ & $326(61.0)$ & $165(62.0)$ \\
\hline Age, years, median (range) & $61.5(27-81)$ & $62.5(39-78)$ & $63.0(27-82)$ & $63.0(27-82)$ \\
\hline \multicolumn{5}{|l|}{ Race, $n(\%)$} \\
\hline Caucasian & $80(100)$ & $32(100)$ & $306(57.3)$ & $155(58.3)$ \\
\hline Asian/Oriental & 0 & 0 & $184(34.5)$ & $94(35.3)$ \\
\hline Other/not collected & 0 & 0 & $44(8.2)$ & $17(6.4)$ \\
\hline \multicolumn{5}{|l|}{ ECOG PS, $n(\%)$} \\
\hline 0 & $24(30.0)$ & $11(34.4)$ & $301(56.4)$ & $147(55.3)$ \\
\hline 1 & $56(70.0)$ & $21(65.6)$ & $233(43.6)$ & $119(44.7)$ \\
\hline \multicolumn{5}{|l|}{$K R A S$ status, ${ }^{\text {a }} n(\%)$} \\
\hline Wild type & $35(43.8)$ & $17(53.1)$ & $262(49.1)$ & $131(49.2)$ \\
\hline Mutant & $45(56.3)$ & $15(46.9)$ & $272(50.9)$ & $135(50.8)$ \\
\hline \multicolumn{5}{|l|}{ Time since diagnosis of first metastasis, ${ }^{\mathrm{a}} n(\%)$} \\
\hline$<18$ months & $16(20.0)$ & 7 (21.9) & $111(20.8)$ & $55(20.7)$ \\
\hline$\geq 18$ months & $64(80.0)$ & $25(78.1)$ & $423(79.2)$ & $211(79.3)$ \\
\hline Time from initial diagnosis, months, mean (SD) & $42.4(24.7)$ & $46.6(34.7)$ & $44.1(29.3)$ & $45.5(28.3)$ \\
\hline \multicolumn{5}{|l|}{ Primary tumor site, $n(\%)$} \\
\hline Colon & $49(61.3)$ & $23(71.9)$ & $338(63.3)$ & $161(60.5)$ \\
\hline Rectal & $31(38.8)$ & $9(28.1)$ & $196(36.7)$ & $105(39.5)$ \\
\hline \multicolumn{5}{|l|}{ Number of organ sites involved, $n(\%)^{\mathrm{b}}$} \\
\hline $1-2$ & $51(64)$ & $19(59)$ & $309(58)$ & $146(55)$ \\
\hline$\geq 3$ & $29(36)$ & $13(41)$ & $225(42)$ & $120(45)$ \\
\hline \multicolumn{5}{|l|}{ Number of prior regimens, ${ }^{\mathrm{c}} n(\%)$} \\
\hline 1 & 0 & 0 & 0 & 0 \\
\hline 2 & $15(18.8)$ & 7 (21.9) & $95(17.8)$ & $45(16.9)$ \\
\hline 3 & $16(20.0)$ & 7 (21.9) & $119(22.3)$ & $54(20.3)$ \\
\hline$\geq 4$ & $49(61.3)$ & $18(56.3)$ & $320(59.9)$ & $167(62.8)$ \\
\hline \multicolumn{5}{|l|}{ All prior systemic cancer therapeutic agents, ${ }^{\mathrm{c}, \mathrm{d}} n(\%)$} \\
\hline Bevacizumab & $80(100.0)$ & $31(96.9)$ & $534(100)$ & $265(99.6)$ \\
\hline Cetuximab/panitumumab & $41(51.3)$ & $19(59.4)$ & $278(52.1)$ & $144(54.1)$ \\
\hline Fluoropyrimidine $^{\mathrm{e}}$ & $80(100.0)$ & $32(100.0)$ & $534(100)$ & $266(100)$ \\
\hline Irinotecan & $80(100.0)$ & $32(100.0)$ & $534(100)$ & $266(100)$ \\
\hline Oxaliplatin & $80(100.0)$ & $32(100.0)$ & $534(100)$ & $266(100)$ \\
\hline Regorafenib & $20(25.0)$ & $9(28.1)$ & $91(17)$ & $53(19.9)$ \\
\hline Other & $73(91.3)$ & $30(93.8)$ & $471(88.2)$ & $237(89.1)$ \\
\hline Refractory to fluoropyrimidines, ${ }^{\mathrm{f}} n(\%)$ & $72(90.0)$ & $31(96.9)$ & $497(93.1)$ & $240(90.2)$ \\
\hline
\end{tabular}

ECOG PS, Eastern Cooperative Oncology Group performance status; SD, standard deviation

a As randomized

${ }^{b}$ Based on the number of unique lesion sites (organs) per patient

${ }^{c}$ Includes all prior systemic therapies (neoadjuvant, adjuvant, metastatic)

${ }^{\mathrm{d}}$ Patients with multiple levels are counted in each applicable category

e Fluoropyrimidine includes 5-FU-containing agents fluorouracil, capecitabine, doxifluridine, S-1, tegafur, and UFT

${ }^{\mathrm{f}}$ Refractory the last time fluoropyrimidine was part of the regimen. Refractory defined as: regimens with radiologic progression $\leq 93$ days from the last dose of the last component of the regimen for regimens intended to treat metastatic disease (or of missing intent), and with radiologic progression $\leq 186$ days for adjuvant/neoadjuvant regimens 
A

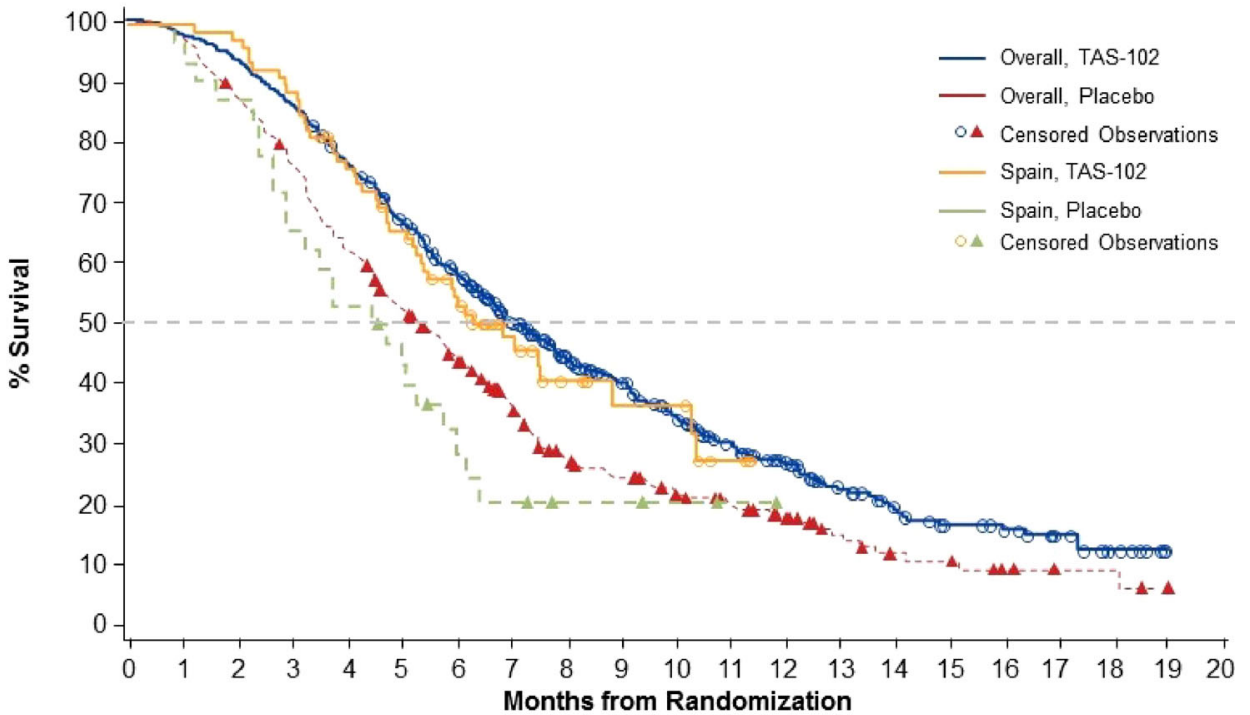

$\mathrm{N}$ at Risk:

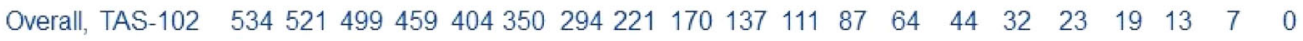

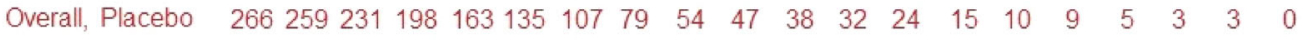

Spain, TAS-102 $\quad 80 \begin{array}{lllllllllllll}80 & 78 & 71 & 59 & 50 & 36 & 21 & 14 & 9 & 9 & 4 & 0\end{array}$

Spain, Placebo

$\begin{array}{lllllllllllll}32 & 31 & 28 & 21 & 17 & 13 & 7 & 5 & 3 & 3 & 2 & 1 & 0\end{array}$

B

\begin{tabular}{lcccc}
\cline { 2 - 5 } B & \multicolumn{3}{c}{ Spanish Population } & \multicolumn{2}{c}{ Overall Population } \\
\cline { 2 - 5 } Month & TAS-102, \% (95\% Cl) & Placebo, \% (95\% CI) & TAS-102, \% (95\% Cl) & Placebo, \% (95\% CI) \\
3 & $88.8(79.5-94.0)$ & $65.6(46.6-79.3)$ & $86.0(82.7-88.6)$ & $75.1(69.4-79.9)$ \\
6 & $53.2(41.3-63.7)$ & $28.5(13.7-45.3)$ & $57.8(53.5-61.9)$ & $43.5(37.4-49.4)$ \\
9 & $36.6(23.4-49.9)$ & $20.4(7.9-36.9)$ & $40.1(35.6-44.6)$ & $24.2(18.9-29.9)$ \\
12 & $27.5(14.0-42.9)$ & $20.4(7.9-36.9)$ & $26.6(22.2-31.1)$ & $17.6(12.7-23.1)$
\end{tabular}

Fig. 2 a Kaplan-Meier curves for overall survival in the Spanish subgroup and overall RECOURSE populations; $\mathbf{b}$ overall survival estimates for the TAS-102 and placebo treatment groups at months 3, 6, 9, and 12

Table 2 Overall survival and progression-free survival for the Spanish subgroup and overall RECOURSE population (ITT population) [11]

\begin{tabular}{llllll}
\hline & \multicolumn{2}{l}{ Spanish subgroup } & & \multicolumn{2}{l}{ Overall population } \\
\cline { 2 - 3 } & TAS-102 $(n=80)$ & Placebo $(n=32)$ & & TAS-102 $(n=534)$ & Placebo $(n=266)$ \\
\hline Median OS, months & 6.8 & 4.6 & 7.1 & 5.3 \\
HR (95 \% CI) & $0.47(0.28-0.78)$ & & $0.68(0.58-0.81)$ & \\
$P$ value & $P=0.0032$ & & $P<0.001$ & 1.7 \\
Median PFS, months & 2.0 & 1.7 & 2.0 & \\
HR (95 \% CI) & $0.47(0.30-0.74)$ & & $0.48(0.41-0.57)$ & \\
$P$ value & $P=0.001$ & & $P<0.0001$ & \\
\hline
\end{tabular}

CI confidence interval, $H R$ hazard ratio, ITT intention to treat, $O S$ overall survival, $P F S$ progression-free survival

and, therefore, a worse prognosis. The Kaplan-Meier curves are largely coincident for the TAS-102 arms in Spanish patients and overall (Fig. 2). There was no significant benefit in favor of TAS-102 in terms of best overall response and disease control rates, although both showed trends favoring TAS-102. Similar results were seen for the Phase 3 trial of regorafenib which noted similar OS and PFS for Japanase and primarily Caucasian non-Japanese patients [13]. A recent analysis of European cancer registries showed that the mean 5-year OS for colorectal and 


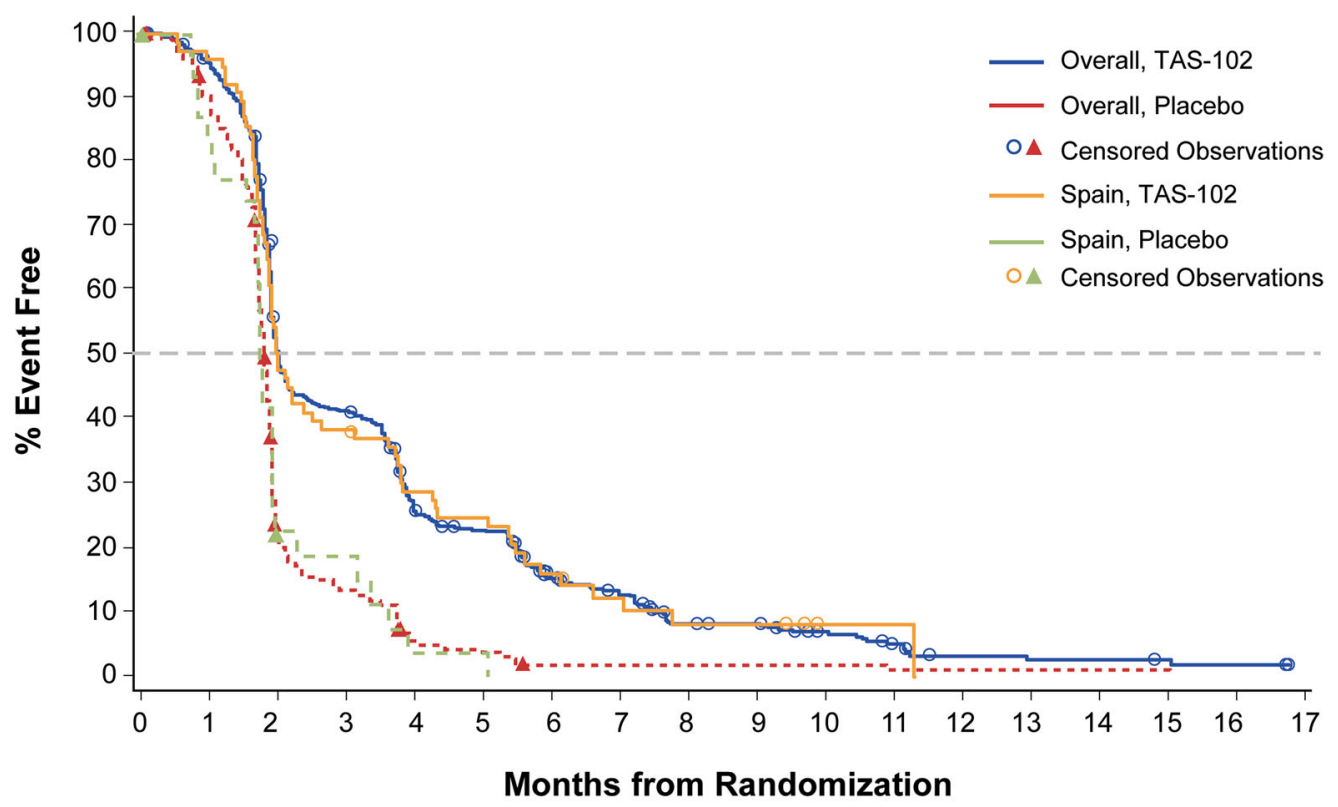

N at Risk:

$\begin{array}{lcccccccccccccccccc}\text { Overall, TAS-102 } & 534 & 488 & 238 & 205 & 121 & 107 & 66 & 52 & 30 & 26 & 18 & 11 & 5 & 4 & 4 & 2 & 2 & 0 \\ \text { Overall, Placebo } & 266 & 227 & 51 & 32 & 10 & 7 & 2 & 2 & 2 & 2 & 2 & 1 & 1 & 1 & 1 & 0 & & \\ \text { Spain, TAS-102 } & 80 & 74 & 36 & 28 & 21 & 17 & 9 & 5 & 4 & 4 & 1 & 1 & 0 & & & & \\ \text { Spain, Placebo } & 32 & 25 & 6 & 5 & 1 & 0 & & & & & & & & & & & \end{array}$

Fig. 3 Kaplan-Meier curves for radiologic progression-free survival in the Spanish subgroup and overall RECOURSE populations

rectal cancers in Spanish patients was similar to the European mean for those cancers, with mean OS in Southern Europe being similar to that of Northern and Central Europe, and greater than that in the UK, Ireland, and Eastern Europe [14]. This suggests that survival for $\mathrm{mCRC}$ in Spanish patients is representative of Europe as a whole and that the results presented here corroborate results for the whole continent [15].

There were a number of differences in the baseline characteristics of the Spanish population when compared with the overall RECOURSE population, as might be expected in a multicenter international study that included patients from Europe, Asia, the United States, and Australia. All of the patients from Spain were Caucasian, compared with $57.6 \%$ of the overall population $(34.8 \%$ of the patients in the trial were Asian). The demonstrated efficacy of TAS-102 in Caucasian Spanish patients is encouraging for the generalizability of the results of the RECOURSE study to different populations. This is significant because the phase 2 trial of TAS-102 in mCRC was conducted solely in Japanese patients [9], and differences in response have been noted previously between Western and Japanese subjects for gefitinib in lung cancer [16]. In the prespecified analysis of regional subgroups in RECOURSE, the OS difference between TAS-102 and placebo groups in the Japanese population (HR: 0.75, $95 \%$ CI: 0.57-1.00) was not as pronounced as in the US (HR:
0.56, $95 \%$ CI: 0.34-0.94) or European (HR: 0.62, $95 \%$ CI: $0.48-0.80)$ populations, which were largely composed of Caucasian patients. Demographics as well as the small size of the Spanish subgroup relative to the overall study may explain the apparent survival benefit in the Spanish population.

There was some imbalance of colon cancer versus rectal cancer in the placebo group enrolled in Spain, compared with the TAS-102 arm and the overall population. Although patients in the Spanish subgroup had similar time from diagnosis, number of rounds of prior chemotherapy, and KRAS mutation prevalence as the overall RECOURSE population, the Spanish subpopulation had $50 \%$ higher incidence of ECOG performance status of 1 than the overall study population, which indicates poorer health status. This higher incidence of a worse ECOG performance status would be expected to have a negative impact on outcomes since performance status on its own has been shown to be a good prognostic factor for patients with advanced cancer [17]. Despite this, the time to deterioration to performance status $\geq 2$ in Spanish patients was similar to that observed in the overall population for both TAS-102 (5.4 months versus 5.7 months) and placebo (3.3 vs 4.0 months) [11]. The reasons for this discrepancy in the ECOG performance status of Spanish patients and the overall population are unclear. However, they are consistent with a similar analysis of the Phase 3 CORRECT trial 
Table 3 Adverse events in the Spanish subgroup and overall RECOURSE population (as-treated population) [11]

\begin{tabular}{|c|c|c|c|c|}
\hline & \multicolumn{2}{|l|}{ Spanish population } & \multicolumn{2}{|l|}{ Overall population } \\
\hline & TAS-102 $(n=80)^{\mathrm{a}}$ & Placebo $(n=32)^{\mathrm{a}}$ & TAS-102 $(n=533)^{\mathrm{a}}$ & Placebo $(n=265)^{\mathrm{a}}$ \\
\hline Any $\mathrm{AE}, n(\%)$ & $80(100)$ & $31(97)$ & $524(98)$ & $247(93)$ \\
\hline Grade $\geq 3$ AEs, $n(\%)$ & $58(73)$ & $18(56)$ & $370(69)$ & $137(52)$ \\
\hline \multicolumn{5}{|c|}{ Most common AEs, any grade ( $\geq 20 \%$ in TAS-102 group), $n(\%)$} \\
\hline Nausea & $31(39)$ & $5(16)$ & $258(48)$ & $63(24)$ \\
\hline Vomiting & $20(25)$ & $3(9)$ & $148(28)$ & $38(14)$ \\
\hline Decreased appetite & $29(36)$ & $10(31)$ & $208(39)$ & $78(29)$ \\
\hline Fatigue & $11(14)$ & $3(9)$ & $188(35)$ & $62(23)$ \\
\hline Diarrhea & $24(30)$ & $5(16)$ & $170(32)$ & $33(13)$ \\
\hline Abdominal pain & $13(16)$ & $3(9)$ & $79(15)$ & $36(14)$ \\
\hline Asthenia & $49(61)$ & $13(41)$ & $97(18)$ & $30(11)$ \\
\hline Mucosal inflammation & $18(23)$ & $8(25)$ & $30(5.6)$ & $12(4.5)$ \\
\hline \multicolumn{5}{|c|}{ Most common grade $\geq 3$ AEs ( $\geq 5 \%$ in TAS-102 group), $n$ ( $\%$ ) } \\
\hline Asthenia & $10(13)$ & $2(6)$ & $18(3)$ & $8(3)$ \\
\hline Back pain & $4(5)$ & $1(3)$ & $9(2)$ & $2(1)$ \\
\hline \multicolumn{5}{|c|}{ Grade $\geq 3$ laboratory abnormalities that worsened by baseline $\geq 1$ grade, ${ }^{\mathrm{a}} n(\%)$} \\
\hline Neutropenia & $32(40)$ & 0 & $200 / 528(38)$ & 0 \\
\hline Leukopenia & $13(16)$ & 0 & $113 / 528(21)$ & 0 \\
\hline Lymphocytopenia & 8/76 (11) & $2 / 31(7)$ & $112 / 522(21)$ & $26 / 262(10)$ \\
\hline Anemia & $11(14)$ & $2(6)$ & $96 / 528(18)^{\mathrm{b}}$ & $8 / 263(3)$ \\
\hline Increased total bilirubin & $7(8)$ & $6(19)$ & $45 / 526(9)$ & $31 / 262(12)$ \\
\hline Increased alkaline phosphatase & $5(6)$ & $6 / 31(19)$ & $42 / 526(8)$ & $28 / 262(11)$ \\
\hline Thrombocytopenia & $2(3)$ & 0 & $27 / 528(5)$ & $1 / 263(<1)$ \\
\hline Serious AEs & $16(20)$ & $12(38)$ & $158(30)$ & $89(34)$ \\
\hline Hospitalizations, $n(\%)$ & $16(20)$ & $12(38)$ & $165(31)$ & $96(36)$ \\
\hline Due to serious $\mathrm{AE}$ & $14 / 16(88)$ & $12 / 12(100)$ & $140 / 165(85)$ & $85 / 96(89)$ \\
\hline Due to febrile neutropenia & $1 / 16(6)$ & 0 & $14 / 165(9)$ & 0 \\
\hline Due to health deterioration & 0 & $1 / 12(8)$ & $15 / 165(9)$ & 9/96 (9) \\
\hline
\end{tabular}

a The total as-treated population serves as denominator unless otherwise indicated

b One patient was diagnosed with Grade 4 anemia

$A E$ adverse event

of regorafenib which noted a higher rate of ECOG performance status of 0 among Japanese patients compared to the primarily Caucasian non-Japanese population [13]. It is possible, therefore, that regional or cultural differences in interpretation of ECOG performance status definitions had a role.

TAS-102 was generally well tolerated in this subgroup of patients from the RECOURSE study, with the overall safety profile being similar to that of the overall population. Importantly, no new safety signals were seen in this Spanish population. As with the overall study, neutropenia was the most frequently observed, clinically meaningful laboratory abnormality or adverse event, with one patient being hospitalized for febrile neutropenia. The only substantial difference reported in the safety profile of TAS-102 between the Spanish and overall RECOURSE populations was a higher incidence of asthenia and lower incidence of fatigue in Spanish patients. This difference is likely due to subtle local differences in interpretation of the definition of these two overlapping adverse events [18]. The rate of serious adverse events and hospitalizations in the Spanish subgroup was somewhat lower than in the overall population, but this could merely reflect local differences in healthcare expenditure, socioeconomic status, lifestyle, and general health status of the population [14], as well as the relatively low number of patients in this cohort. The lack of difference in adverse events differs from that seen in the recent trial of regorafenib, which noted a higher incidence of certain adverse events among Japanese patients than non-Japanese patients-including a higher incidence of Grade 3 adverse events [13]. 
The data presented here are limited by the fact that this was an unplanned post hoc analysis of the subgroup of patients who were randomized in Spain. As such, this analysis may not have had sufficient statistical power to draw definitive conclusions about the efficacy of TAS-102. However, this population represents a substantial proportion of the European geographic region, an analysis of which was preplanned. Furthermore, the Spanish population was larger than the American and Australian geographic subregions for which there were preplanned analyses.

The results of this analysis of a subgroup of Spanish patients with mCRC refractory to standard therapies who were enrolled in the international, multicenter, randomized, placebo-controlled RECOURSE study indicate that the results of the larger trial are generally applicable in this subpopulation. TAS-102 was found to impart significant improvements in OS and PFS compared with placebo, similar to that seen in the overall trial. TAS-102 was generally well tolerated in this population of Spanish patients, with no new safety signals unique to this particular population identified. TAS-102 may be a good treatment option for Spanish patients with mCRC who are refractory to standard treatments.

Acknowledgments The authors were responsible for all content and editorial decisions and received no honoraria related to the development of this publication. All authors contributed to the research, writing, and reviewing of all drafts of this manuscript. All authors approved the final draft. Editorial support in the preparation of this publication was provided by Phase Five Communications, supported by Taiho Oncology Inc.

\section{Compliance with ethical standards}

Conflict of interest Author A reports employment at Taiho Oncology Inc. Author B reports providing scientific advice to Bayer. Author $\mathrm{C}$ reports consulting/advisory fees from Amgen, Boehringer Ingelheim, Celgene, Chugai, Imclone Systems Inc., Eli Lilly and Company, Merck \& Co., Merck Serono, Millennium, Novartis, F. Hoffmann-La Roche Ltd., Sanofi, Symphogen, and Taiho Oncology Inc. All other authors declare that they have no conflicts of interest.

Informed consent Informed consent was obtained from all individual participants included in the study.

Research involving human participants All procedures performed in studies involving human participants were in accordance with the ethical standards of the institutional and/or national research committee and with the 1964 Helsinki declaration and its later amendments or comparable ethical standards.

\section{References}

1. Emura T, Murakami Y, Nakagawa F, Fukushima M, Kitazato K. A novel antimetabolite, TAS-102 retains its effect on FU-related resistant cancer cells. Int J Med. 2004;13(4):545-9.

2. Tanaka N, Sakamoto K, Okabe H, Fujioka A, Yamamura K, Nakagawa F, et al. Repeated oral dosing of TAS-102 confers high trifluridine incorporation into DNA and sustained antitumor activity in mouse models. Oncol Rep. 2014:32(6):2319-26. doi:10.3892/or.2014.3487.

3. Nukatsuka M, Nakagawa F, Saito H, Sakata M, Uchida J, Takechi T. Efficacy of combination chemotherapy using a novel oral chemotherapeutic agent, TAS102 , with irinotecan hydrochloride on human colorectal and gastric cancer xenografts. Anticancer Res. 2015;35(3):1437-45.

4. Emura T, Suzuki N, Fujioka A, Ohshimo H, Fukushima M. Potentiation of the antitumor activity of alpha, alpha, alpha-trifluorothymidine by the co-administration of an inhibitor of thymidine phosphorylase at a suitable molar ratio in vivo. Int J Oncol. 2005;27(2):449-55.

5. Meyerhardt JA, Mayer RJ. Systemic therapy for colorectal cancer. N Engl J Med. 2005;352(5):476-87. doi:10.1056/NEJMra040958.

6. Overman MJ, Kopetz S, Varadhachary G, Fukushima M, Kuwata K, Mita A, et al. Phase I clinical study of three times a day oral administration of TAS-102 in patients with solid tumors. Cancer Invest. 2008;26(8):794-9. doi:10.1080/ 07357900802087242 .

7. Overman MJ, Varadhachary G, Kopetz S, Thomas MB, Fukushima M, Kuwata $\mathrm{K}$, et al. Phase 1 study of TAS-102 administered once daily on a 5-day-per-week schedule in patients with solid tumors. Invest New Drugs. 2008;26(5):445-54. doi:10.1007/s10637-008-9142-3.

8. Doi T, Ohtsu A, Yoshino T, Boku N, Onozawa Y, Fukutomi A, et al. Phase I study of TAS-102 treatment in Japanese patients with advanced solid tumours. Br J Cancer. 2012;107(3):429-34. doi:10.1038/bjc.2012.274.

9. Yoshino T, Mizunuma N, Yamazaki K, Nishina T, Komatsu Y, Baba H, et al. TAS-102 monotherapy for pretreated metastatic colorectal cancer: a doubleblind, randomised, placebo-controlled phase 2 trial. Lancet Oncol. 2012;13(10):993-1001. doi:10.1016/S1470-2045(12)70345-5.

10. Bendell JC, Rosen LS, Mayer RJ, Goldman JW, Infante JR, Benedetti F, et al. Phase 1 study of oral TAS-102 in patients with refractory metastatic colorectal cancer. Cancer Chemother Pharmacol. 2015;76(5):925-32. doi:10.1007/s00280015-2850-4.

11. Mayer RJ, Van Cutsem E, Falcone A, Yoshino T, Garcia-Carbonero R, Mizunuma N, et al. RECOURSE Study Group. Randomized trial of TAS-102 for refractory metastatic colorectal cancer. N Engl J Med. 2015;372(20):1909-19. doi:10.1056/NEJMoa1414325.

12. Eisenhauer EA, Therasse P, Bogaerts J, Schwartz LH, Sargent D, Ford R, et al. New response evaluation criteria in solid tumours: revised RECIST guideline (version 1.1). Eur J Cancer. 2009;45(2):228-47. doi:10.1016/j.ejca.2008.10.026.

13. Yoshino T, Komatsu Y, Yamada Y, Yamazaki K, Tsuji A, et al. Randomized phase III trial of regorafenib in metastatic colorectal cancer: analysis of the CORRECT Japanese and non-Japanese subpopulations. Invest New Drugs. 2015;33(3):740-50. doi:10.1007/s10637-014-0154-x Epub 2014 Sep 12.

14. De Angelis R, Sant M, Coleman MP, Francisci S, Baili P, Pierannunzio D, EUROCARE Working Group, et al. Cancer survival in Europe 1999-2007 by country and age: results of EUROCARE-5-a population-based study. Lancet Oncol. 2014;15(1):23-34. doi:10.1016/S1470-2045(13)70546-1.

15. Ohtsu A, Yoshino T, Wabha MM, Benedetti FM, Mayer RJ, van Cutsem E et al; RECOURSE Study Group. Phase 3 RECOURSE trial of TAS-102 versus placebo with best supportive care in patients with metastatic colorectal cancer: geographic subgroups. 2015 American Society of Clinical Oncology Annual Meeting; 29 May-2 June, 2015; Chicago, IL. Abstract no. 3564. http://meet inglibrary.asco.org/content/148025-156. Accessed 4 Feb 2016.

16. Fukuoka M, Yano S, Giaccone G, Tamura T, Nakagawa K, Douillard JY, et al Multi-institutional randomized phase II trial of gefitinib for previously treated patients with advanced non-small-cell lung cancer (The IDEAL 1 Trial) [corrected]. J Clin Oncol. 2003;21(12):2237-46. doi:10.1200/JCO.2003.10.038.

17. Jang RW, Caraiscos VB, Swami N, et al. Simple prognostic model for patients with advanced cancer based on performance status. J Oncol Pract. 2014;10(5):e335-41.

18. Saguil A. Evaluation of the patient with muscle weakness. Am Fam Physician. 2005;71(7):1327-36. 